Bulletin of the Section of Logic

Volume 50/2 (2021), pp. 131-149

https://doi.org/10.18778/0138-0680.2021.07

Federico L. G. Faroldi

\title{
TOWARDS A LOGIC OF VALUE AND DISAGREEMENT VIA IMPRECISE MEASURES
}

\begin{abstract}
After putting forward a formal account of value disagreement via imprecise measures, I develop a logic of value attribution and of (dis)agreement based on (exact) truthmaker semantics.
\end{abstract}

Keywords: Value, disagreement, truthmaker semantics, hyperintensionality.

\section{Introduction}

Suppose you and a friend have different value judgments on a proposition or a state of affairs, where all other relevant considerations are on a par (you both have the same information, same reasoning power, perhaps endorse the same moral theory, etc.): we call such a situation a value-based peer disagreement. Think of two act-utilitarians A and B, with the same background information. A thinks that improving an elephant's life from a circus by closing it down is worth firing all the circus' employees; B doesn't. Both agree on the facts; both agree on the factual consequences, they even agree on the value of improving the elephant's life; their only disagreement is on its value (as measured by the (dis)value if some of the consequences, in this case).

Modulo some minor points, modeling epistemic peer disagreement seems conceivably routine, at least as soon as we agree on how to represent beliefs. This usually involves some probability distribution: for agents $\mathrm{A}$ and $\mathrm{B}$, and proposition $\mathrm{p}$, agents $\mathrm{A}$ and $\mathrm{B}$ disagree over $\mathrm{p}$ when $\mathcal{B}^{A}(p) \neq \mathcal{B}^{B}(p)$, where

Presented by: Tomasz Jarmużek, Fengkui Ju, Piotr Kulicki, Beishui Liao

Received: August 31, 2020

Published online: May 27, 2021

(C) Copyright by Author(s), Łódź 2021

(C) Copyright for this edition by Uniwersytet Łódzki, Łódź 2021 
$\mathcal{B}:$ Agents $\times$ Prop $\rightarrow[0,1]$ is a belief function over propositions indexed to agents. However, there is no such agreement on value disagreement.

The received view, in the contemporary literature, is usually to take a domain of objects (outcomes, events, individuals, etc.) and to order them via agent-based preference relations. After that, one can either compare them directly or represent these qualitative relations as functions into more or less standard (quantitative) structures preserving the ordering. Given certain structural assumptions, one may then perform more complicated operations, like aggregation, taking averages, etc. These accounts are ridden with very well-known problems. Let me mention a few. Preference orderings are almost always used, and they are transitive and complete relations. Both properties are dubious: first, several people have argued that preference (or goodness) is not transitive, as it is shown by case of value parity and sweetening; ${ }^{1}$ second, completeness, at least in the case of value, seems completely unrealistic, if anything, at least for the very fact that there is, reasonably, an infinite number of possibilities, and agent with finite resources will hardly be able to have a definite preference ordering on an infinite number of options. ${ }^{2}$ This objection is plausibly weakened if one adopts a theory according to which value assignments are completely objective. Even in this case, however, a plausible case for incompleteness can be built, given some assumption of uncertainty or intrinsic indeterminacy. Third, not all moral theories are expressible via preference relations (cf. [10]), thus making an account which does not depend on preference relations preferable.

Another concern against preference approaches has to do with the fact that often a quantitative representation into certain number structures, like the real numbers, is sought. This poses two kinds of problems: first, in order to guarantee the existence of a quantitative representation, one often needs to require further structural conditions on the qualitative properties, most of which are unnatural (for an example, think of a "continuity" or "solvability" condition); second, one can think that numbers (or in general, other quantitative structures) are philosophically ill-suited to represent, model or understand values.

In the next section, I will sketch a way to solve some of the aforementioned issues.

\footnotetext{
${ }^{1}$ See for instance [28].

${ }^{2}$ For some work in decision theory without completeness, see [22, 23, 24].
} 


\section{Values and imprecise measures, informally}

In [12], I put forward and defended a formal account of value disagreement via imprecise measure theory. Very briefly, each agent has associated a set of measures that determine an interval (not necessarily numeric). Philosophical consideration in favor of this approach, besides the fact that it bypasses the criticism of standard preference-based approaches, are to be found when one takes into account (i) considerations of normative uncertainty, (ii) considerations of normative indeterminacy, (iii) issues summarized by the label of "transformative experiences". ${ }^{3}$

As for (i), normative uncertainty, suppose you are about to leave for an imminent trip, and your insurance company instructs you to indicate an amount of money to insure your luggage, in case of loss, theft, and so on. What is the right amount of money (in this case) at which you value your belongings? Modulo considerations of factual uncertainty, which will plausibly be taken into account by a probability distribution, you might be unsure how to value different things: that particular sweater your grandmother made for you last Christmas before she died, for instance, is not worth much as a sweater, but is priceless. Or, in a different but possible context, say, if your grandmother were still to live, or after her death you discovered she was a horrible human being, you could instead attach a more definite value. Or again, you might not have any opinion about how valuable something is, at least in come contexts, but not in others, which is again different to say that something has no value (or value 0 , if we use money as linear utilities). An interval or imprecise approach can take care of these considerations.

As for (ii), normative indeterminacy, suppose it is indeterminate that an action is permissible or impermissible, plausibly for Sorites-like reasons. For instance it is permissible to interrupt the focus of a bus driver to ask a question, but it is impermissible to harass them for two hours; however, it seems indeterminate whether asking three or four questions is permissible or not (similar examples abound in the literature). It seems that an interval or imprecise approach can take care of these considerations. In this case, the indeterminacy does not depend on subjective extra-normative circumstances (different people having different informations, for example),

\footnotetext{
${ }^{3} \mathrm{~A}$ referee points out that this account may even be in agreement with research into the mental processes of estimating cardinalities. Cf. [19, 20].
} 
but on a genuine normative gap, as it were. Of course whether this gap is subject-dependent or subject-independent depends on the normative theory in question. Related considerations can be made with regard to parity, a fourth value relation beside 'better than', 'worse than' and 'equal' that some philosophers argue exist. ${ }^{4}$ An interval-based approach can arguably take care of this notion. ${ }^{5}$

As for (iii), "transformative experiences", one can increase or decrease the value of something as a result of thinking ahead. Suppose you value immensely philosophy, or abstract thinking. However, you know that, as you age, your intellectual powers will likely decrease, and as a result you will value much more more practical tasks or human companionship, of the kind that just a family or old friends can provide. A family or old friends cannot be obtained overnight, and this perhaps makes you value abstract thinking a bit less even now, for valuing it so highly will be detrimental to other activities beneficial to your future value set. This can be understood as a case of interpersonal disagreement in the sense of there being disagreement between myself at the present time and myself in the future. Using a diachronic approach (i.e. indexing one's function to time) might not be the right choice in this context; having intervals of value, rather than sharp values, seems a good way to go, for you can adjust upwards or downwards your current value, singly considered, given this kind of updates.

In the present paper, I briefly go through such an account of value disagreement via imprecise measure theory in Sect. 3. In Sect. 4 I develop some ideas leading to a logic of value attribution and of disagreement, based on truthmaker semantics.

\section{Values and imprecise measures, formally}

We suggest that every agent $\alpha \in A$ has associated a set of partial functions from sets of states of affairs to their value. Partiality take care that some things may be incomparable or incommensurable, even intrapersonally.

\footnotetext{
${ }^{4}$ See for one $[6,5,7]$.

${ }^{5}$ For a similar approach to parity, without intervals but with multiple relations, see $[25,26]$.
} 


\subsection{Values and imprecise measures: first pass}

We now define these intuitions more precisely.

Definition 1 (Value space). Let $(\Omega, \mathcal{F}, \mathcal{M}, A)$ be a value space, where

1. $\Omega$ is a set of (partial) states ordered by a partial order $\sqsupseteq$;

2. $\mathcal{F}$ is a suitably generated structure of its subsets;

3. $\mathcal{M}$ is a (finite) set of partial indexed signed measures $\mu: \mathcal{F} \times A \times I \rightarrow$ $\mathbb{R}$ which we write $\mu_{i}^{\alpha}$, for each agent $\alpha \in A$, and $i \in I$ where $I$ is an index set.

4. $A$ is a finite set of agents.

Given the set of states $\Omega$, where the partial order represents parthood, a suitably generated structure $\mathcal{F}$ of its subsets could be its power set if $\Omega$ is finite, a sigma-algebra if not, or a less rich structure. We do not take a stance on this point, except to note that we showed how to construct a measure on a weak structure such as a (join) semilattice in Theorem 13 of [13]. The intuition (which will be made precise later when the logic part is introduced) is that some propositions can be identified with sets of states of affairs (namely, those which verify the proposition).

Although the definition of a value space is quite general, for the immediate purposes of this paper we can ignore point 2, given that for the family $\mathcal{M}^{\alpha}$ of indexed signed measures $\mu_{i}{ }^{6}$ a set $i \in I$ (used to distinguish measures) for each agent $\alpha$, no further requirement is imposed. In particular, the measure can take negative values and it is not normalized. Additionally it is not required to be (either finitely or sigma-) additive, in order to cash out a substantial plausible feature of value structures: first, value judgments may not be additive, for adding something extraneous to a positively valued state of affairs may decrease its value. ${ }^{7}$ One can impose monotonicity as a separate requirement, although the consensus seems to be that value is not monotonic. ${ }^{8}$ Second, we use $\mathbb{R}$ out of convenience. As

\footnotetext{
${ }^{6}$ To avoid clutter, I write $\mu$ or $\mu_{i}$ suppressing the agent or the index, when it is clear from the context.

${ }^{7}$ For non-additive measures, see [29].

${ }^{8}$ By monotonicity in this context we mean the following: for any $s, t \in \Omega$ and $\mu \in \mathcal{M}$, if $s \sqsupseteq t$ then $\mu(s) \geq \mu(t)$.
} 
an alternative, it has been suggested to use $* \mathbb{R}$ in order to represent normative reasons (see [13] and [1]), although the use of non-standard structures in utility theory is well-known. Third, we have not specified a range: for instance one can assign infinite values to the functions in order to account for some particular phenomena, thus adjoining $\{-\infty ; \infty\}$ to the codomain. Fourth, in case one thinks that the set of states $\Omega$ is a set of partial states that can be joined arbitrarily, thus eschewing a possibility requirement and admitting of impossible state of affairs, one would be at loss with a positive and normalized measure. Moreover, one could very well assign positive value to impossible states. Fifth, and finally, one could very well require that $\mu(\emptyset)=0$, although it is not clear whether there is consensus on the fact that the empty event (or state, etc.) has null value, or there may be situations where it may have a non-null value. Thus the term 'measure' is used pretty loosely.

The first advantage to such a set-up, w.r.t. more traditional ones, is that whatever order structure the set of propositions/states of affairs may have, it is not reflected in the value judgments, since the function is not required to be e.g. monotonic. A simple example to illustrate the point at hand. I take measures to be precise. Suppose $s_{1}=$ "Mary gives the first six months of her salary to charity", $s_{2}=$ "gives the last six months of her salary to charity" and $s_{1+2}=s_{1} \sqcup s_{2}=$ "Mary gives the first and last six months of her salary to charity". Further suppose that $\mu\left(s_{1}\right)=5 ; \mu\left(s_{2}\right)=5$. If measures were monotonic, we should e.g. expect that $\mu\left(s_{1+2}\right) \geq \mu\left(s_{1}\right)$ and $\mu\left(s_{1+2}\right) \geq \mu\left(s_{2}\right)$. But it is plausible to hold that $\mu\left(s_{1+2}\right)$ can even get a negative value, for Mary would remain without income that year. A "bigger" state can have a value which is e.g. smaller of the values of its parts.

To jump a bit ahead, before having defined the formal means to account for value disagreement, let's have an informal pass of one possible such notion we the example just given. Suppose agents $\mathrm{A}$ and $\mathrm{B}$ have two measures each: $\mu_{1}^{A}\left(s_{1}\right)=5 ; \mu_{2}^{A}\left(s_{1}\right)=6 ; \mu_{1}^{B}\left(s_{1}\right)=1 ; \mu_{2}^{B}\left(s_{1}\right)=-3$. In this simple example, A values state $s_{1}=[5 ; 6]$, whereas $\mathrm{B}$ values state $s_{1}=[-3 ; 1]$ : there's no overlapping, so $\mathrm{A}$ and $\mathrm{B}$ completely disagree over $s_{1}$.

Note that these measures, for each agent, can be arbitrary. This means that, on a more philosophical level, such an approach can account for the following two questions: first, value disagreement can be explained via dif- 
ferent mechanisms to assign values ${ }^{9}$, rather than presupposing the values are assigned just in one way. A consequentialist, for instance, may want the value functions to be additive, whereas a deontologist will presumably reject additivity. Such a flexibility seems possible, for instance, within the very sophisticated framework developed by [10]: where (almost) all normative theories are characterized in a unitary framework, with various combinations of how the options are understood and which properties the preference functions enjoy. If we accept such an approach, then within our framework we can directly compare the deontologist and the consequentialist, the particularist and the utilitarian. But it does not stop here. Second, in fact, we can very easily extend the present account to the higher-order to model disagreement on value theories themselves, insofar as they can be captured by measure functions, which in the higher-order setting will become the elements of the domain themselves: instead of having first-order measures which takes as arguments states of affairs, like in the framework presented so far, we can have second- (and higher-, potentially) order measures which takes as arguments first-order measures. This makes the present account highly general, but we leave the exploration of this issue to further work.

Let's define a couple of other notions necessary to get a grip on different kinds of disagreement.

With an abuse of notation, for every "proposition" ${ }^{10} p$, we define:

Definition 2 (Lower value). Let the lower value of any $p$ be $\underline{M}(p)=$ $\inf \left\{\mu_{i}^{\alpha}(p): \mu_{i}^{\alpha} \in \mathcal{M}\right.$ for all $\alpha \in A$ and $\left.i \in I\right\}$.

Definition 3 (Upper value). Let the upper value of any $p$ be $\bar{M}(p)=$ $\sup \left\{\mu_{i}^{\alpha}(p): \mu_{i}^{\alpha} \in \mathcal{M}\right.$ for all $\alpha \in A$ and $\left.i \in I\right\}$.

DEFinition 4 (Agent-relative Value). Let the agent-relative lower value of any $p$ for agent $\alpha \in A$ be $\bar{M}^{\alpha}(p)=\inf \left\{\mu_{i}^{\alpha}(p): \mu_{i}^{\alpha} \in \mathcal{M}\right.$ for $\alpha \in A$ and all $i \in I\}$. Similarly for the upper value.

It is important to note that contrary to upper and lower probabilities from imprecise probability theory, we do not require that lower and upper value be conjugate, i.e. we do not require that $\underline{M}(p)=1-\bar{M}(p)$.

\footnotetext{
${ }^{9}$ When I use the phrase "assign value", one need not read in it a Nietzschean, as it were, value creation. Such a phrase is meant to be neutral, and can be read also as a merely passive endeavor, that is, simply understanding or feeling what the values already are.

${ }^{10}$ For the purposes of this work, we call proposition or state of affairs any set of states, with all due caveats that should be made precise.
} 
Apart from substantial considerations about values, this follows among other things from the fact that no normalization of measures was imposed.

If we want to keep the analogy with imprecise probability theory, each agent has a precise value measure, but the value of a proposition is an interval. However, I find it much more congenial to the issue of value disagreement to associate to each agent an interval. Technically speaking, this can be done by simply considering that each agent has a family of measures associate to it and by taking the lower and upper value for each agent. With an abuse of notation we can now say that each (second-order, as it were) measure outputs an interval for each agent. ${ }^{11}$

We can now define the following notions.

Definition 5 (Imprecise value space). Let $(\Omega, \mathcal{F}, \mathcal{M}, \underline{M}, \bar{M}, A)$ be an imprecise value space.

Then we can make precise the usual understanding of (sharp) agreement and disagreement in the obvious way, depending on whether the (dis)agreement is just on some propositions (and it is therefore partial) or on all propositions (and it is therefore total).

We define more precisely only one of the several notions one can focus on, because it will be useful later in the logic part:

Definition 6 (Partial Imprecise Peer weak disagreement). Let $(\Omega, \mathcal{F}, \mathcal{M}$, $\underline{M}, \bar{M}, A)$ be an imprecise value space. Agents $\alpha_{1}, \ldots \alpha_{n} \in A$ are in partial imprecise weak disagreement if for some $p \in \mathcal{F}, \bigcap_{\alpha_{i} \in A, i \in \mathbb{N}}\left[\underline{M}^{\alpha_{i}}(p), \bar{M}^{\alpha_{i}}(p)\right]$ $\neq \emptyset$.

We can now define, for every proposition/state of affairs $p$ :

Definition 7 (Imprecise interval value). Let the imprecise interval value $V(p)$ of any $p$ be $V(p)=[\underline{M}(p) ; \bar{M}(p)]$.

This way of modeling value-based peer disagreement is in some continuity with some recent approaches to epistemic peer disagreement (cf. [11]), although the proposed solution to epistemic peer disagreement does not seem to transfer well to the axiological domain, mostly because certain

${ }^{11}$ Somewhat in line with similar ideas in the literature: with intervals (in a variety of manners [16], later revised in [17] and [5]), vectors ([3]), and sets of functions ([18]). 
structural properties of belief functions cannot be assumed for value functions, and these properties are crucial in the epistemic domain and in the solutions developed.

\section{Logics of value and disagreement}

\subsection{Generalizing beyond numbers}

Suppose you do not like, for various philosophical reasons, the idea that values of states of affairs, actions, propositions are to be modeled or expressed with numbers (regardless of whether the target set is something very simple like the natural numbers moral philosophers often employ, or something more usual like the real numbers economist usually employ, or more exotic number structures like hyperreal numbers).

As I hinted in a preceding section, the exact nature of the target set is immaterial for the purposes of this paper; but more than that: it turns out one can generalize the ideas we sketched above to something which should be adequate to a number of different conceptualizations. In particular, we are going to generalize the notion of interval by employing the notion of open set.

Definition 8 (General value space). Let $(\Omega, \mathcal{F}, \mathcal{M}, X)$ be a general value space, where

1. $\Omega$ is a set of (partial) states;

2. $\mathcal{F}$ is a suitably generated structure of its subsets;

3. $\mathcal{M}$ is a family of partial indexed signed measures $\mu_{i}, i \in I$, s.t. $\mu_{i}$ : $\mathcal{F} \rightarrow T$

where $(\Omega, \mathcal{F})$ is like above, and $T$ is a family of subsets of $X$ such that

1. $X \in T, \emptyset \in T$;

2. $\left\{O_{i}\right\}_{i \in I} \subseteq T \Rightarrow \bigcup_{i \in I} O_{i} \in T$ and

3. $\left\{O_{i}^{n}\right\}_{i=1} \subseteq T \Rightarrow \bigcap_{i=1}^{n} O_{i} \in T$.

In other words $T$ is a topology on $X$, which can be for instance a set of actions (we leave open at this point whether $X$ is a subset of $\Omega$ ). 
The underlying philosophical intuitions is that the measure of much you value something is given by the actions you are prepared to perform to preserve or promote it, or some such notion provided by your background metaethical theory, or the things you are prepared give up for it. We can now reformulate all the preceding definitions in terms of open sets, perhaps adjusting one requirement in the following way: $\mu \emptyset=\emptyset$, if the target set is not $\mathbb{R}$.

We can now reformulate all the preceding definitions in terms of open sets, perhaps adjusting one requirement in the following way: $\mu \emptyset=\emptyset$, if the target set is not $\mathbb{R}$. The obvious next step is to use the same topological ideas as above to define a logic of value attribution.

\subsection{First pass: topological semantics}

Given the generalization of the preceding section to open sets, we can now define the topological semantics for the modal logic of value in the obvious way, i.e. where $\square$ is in the interior operator on the topological space. This modal logic, which corresponds to the modal logic $\mathbf{S} \mathbf{4}$, is sound and complete w.r.t. a dense-in-itself metric space (these are well-known standard results).

Definition 9. Let $\mathcal{M}$ be a topological value model $\mathcal{M}=(\tau, v)$ where $\tau$ is a topology and $v: \operatorname{Prop} \rightarrow \mathcal{P}(\mathbb{R})$ is a valuation function from atoms in the language to sets of real numbers. We can define a notion of verification starting from $x=$ iff $x \in v(p)$ and extending it to boolean cases as usual.

Definition 10. Given $v$, let $[\phi]$ be $\{x: x \in \mathbb{R}$ and $x \models \phi\}$. Then $\square_{\alpha} \phi$ (with $\alpha \in A$ an agent) is true if it is in the interior of the set defined by $p$ (via the measure $\mu_{\alpha}$ ):

$x=\square_{\alpha} \phi$ iff $\exists U \in \tau, x \in U$ and $\forall y \in U, y=\phi$, i.e. $\left[\square_{\alpha} \phi\right]=i n t[\phi]$.

I propose to informally interpret in such a logic formulas like $\square_{\alpha} p$ as " $\alpha$ values that $p$ ", since the underlying topological intuition is that $p$ is true in all the open sets, which in our case are the collection of the open intervals of values.

However, such an approach is not only cumbersome, but also not quite satisfactory, as it forgets, as it were, the original measures, and flattens the distinctions, the availability of a metric notwithstanding.

It is clear that this is not a logic of disagreement, but rather of value attribution. 
Be as it may, let's check the plausibility of S4 axioms in the intended interpretation.

Axiom $\mathrm{K}: \square(p \rightarrow q) \rightarrow \square p \rightarrow \square q$ is intuitively adequate, modulo doubts we may have about the material conditional.

Axiom 4: $\square p \rightarrow \square \square p$, i.e. that one values one's valuation seems if not immediate at least acceptable, although with some caution.

Axiom $\mathrm{T}: \square p \rightarrow p$ seems completely unjustifiable: there is no reason for the fact that if something has value also needs to be the case.

However, given the topological facts on the interior operator and on open sets, this is unavoidable in all topological semantics.

Topological semantics (and the corresponding modal logic), therefore, does not seem adequate to model properly our intuitions about the logic of value (and a fortiori, about the logic of value disagreement).

We now go on to define a hyperintensional logic of value on general value spaces that improves on these topological ideas.

\subsection{Logics of value and agreement: hyperintensional logics and semantics}

In this section we present hyperintensional logics of value and agreement. Roughly, we can understand a context as hyperintensional if it draws distinction which are finer-grained than simple logical or necessary equivalence. ${ }^{12}$ For instance, the above $\mathrm{S} 4$ logic is not hyperintensional, in that if agent $A$ values that $p$, and $q$ is logically equivalent to $p$, then automatically agent $\mathrm{A}$ also values that $\mathrm{q}$.

More generally, we can split a definition of hyperintensional by taking into account either necessity or logical equivalence. So we can say that a sentential context $\mathbf{C}$ is non-intensional iff for every sentence $\alpha$ and $\beta$ :

$$
\text { NON-INTENSIONAL: } \nvdash \square(\alpha \equiv \beta) \supset \square(\mathbf{C}(\alpha) \equiv \mathbf{C}(\beta)) \text {. }
$$

A different concept is that of congruentiality. It is obtained when we substitute material equivalence with logical equivalence. In general, we can say that a sentential context $\mathbf{C}$ is congruential iff for every sentence $\alpha$ and $\beta$ :

${ }^{12}$ The term 'hyperintensionality' was proposed by Cresswell some forty years ago (cf. [9]), with reference to logical equivalence. For other early contributions to the topic, see e.g. [27]. [4] is a good introduction to the topic. 
CONGRUENTIAL: If $\vdash \alpha \equiv \beta$ then $\vdash \mathbf{C}(\alpha) \equiv \mathbf{C}(\beta)$.

For the purposes of this paper, we take hyperintensionality to cover congruentiality.

Why go the hyperintensional route when it comes to logics of value, then? There are at least two reasons why the background logic here proposed is hyperintensional: first and more specifically, there is the thought that in order to really see whether there is genuine value disagreement, we must have a grip on the exact meaning of what we are considering. While classical logic is just good up to (classical) logical equivalence, it is widely agreed that meaning is finer-grained than just truth, or even necessary truth. There is partial consensus that hyperintensionality is a good way to track meaning, and exact truthmaker semantics a good way to to make these hyperintensional ideas precise. ${ }^{13}$ Second and more generally, such a logic usually come with the option of being paracomplete, paraconsistent, or both. Having all these options open, i.e. depending on the concrete assignement of verifiers and falsifiers, seems to be the right choice for a logical approach, insofar as it should be general enough to be compatible with different philosophical options to model value incomparability. One prominent position on value incomparability, for instance, takes it to be the case that it should be understood and modeled as a failure of bivalence. ${ }^{14}$

In what follows I sketch a hyperintensional logic of value and disagreement based on truthmaker semantics.

Definition 11 (Value space). Let $(\Omega, S, \mathcal{M})$ be a value space, where

1. $\Omega$ is a set of (partial) states;

2. $S$ is a suitably generated structure of its subsets;

3. $\mathcal{M}$ is a (finite) family of partial indexed signed measures $\mu_{i}^{\alpha}, i \in I$ is an index, $\alpha \in A$ is an agent, s.t. $\mu_{i}^{\alpha}: S \rightarrow 2^{S}$.

The measures are not required to be complete. We can build a model based on a value space in the following way:

${ }^{13}$ I defend a general hyperintensional approach to normative and evaluative phenomena at length in [13]. However, even if one does not find the hyperintensionality route appealing, truthmaker semantics is flexible enough to work with coarser-grained logics, as shown in [30] and [21], for instance.

${ }^{14}$ For a recap on this debate, see [8]. 
Definition 12 (Value space model). Let $(\Omega, S, \preceq, \llbracket \mathbb{\llbracket}, \mathcal{M})$ be a value space model, where

1. $\Omega$ and $\mathcal{M}$ are as above;

2. $(S, \preceq, \llbracket \llbracket)$ is a state space in the exact truthmaker sense, that is:

(a) $S$ is the non-empty set of states;

(b) $\preceq$ is a partial order to be interpreted intuitively as the parthood relation, ie reflexive, transitive, and antisymmetric, and it's upcomplete: we can therefore get a join operation in the usual way: $a \sqcup b=b$ iff $a \preceq b ;$

(c) $\llbracket \rrbracket$ is the valuation function such that $\llbracket p \rrbracket^{+}$is the set of verifiers of $p$, and $\llbracket p \rrbracket^{-}$is the set of falsifiers of $p$, i.e. partial functions from atoms to non-empty subsets of $S$.

Here are the standard clauses for an arbitrary formula to be exactly verified (falsified) by a certain state defined by simultaneous double induction:

DEFinition 4.1 (Exact verification (falsification)).

1. $s \Vdash p$ iff $s \in \llbracket p \rrbracket^{+}$;

2. $s-\| p$ iff $s \in \llbracket p \rrbracket^{-}$;

3. $s \Vdash \neg A$ iff $s \dashv A$;

4. $s \dashv \neg A$ iff $s \| A$;

5. $s \Vdash A \wedge B$ iff for some $s^{\prime}$ and $s^{\prime \prime}, s=s^{\prime} \sqcup s^{\prime \prime}, s^{\prime} \Vdash A$ and $s^{\prime \prime} \Vdash B$;

6. $s-A \wedge B$ iff $s-A$ or $s-B$;

7. $s \Vdash A \vee B$ iff $s \Vdash A$ or $s \Vdash B$;

8. $s-A \vee B$ iff for some $s^{\prime}$ and $s^{\prime \prime}, s=s^{\prime} \sqcup s^{\prime \prime}, s^{\prime}-\| A$ and $s^{\prime \prime}-\| B$

A formula $A \approx_{H} B$ holds in a model when $\llbracket A \rrbracket^{+}=\llbracket B \rrbracket^{+}$, i.e. when $A$ and $B$ have the same verifiers. A formula $B$ is a consequence of a formula $A$, i.e. $A \models_{H D L} B$, iff $\llbracket A \rrbracket^{+} \succeq \llbracket B \rrbracket^{+}$. 
The usual notions are pretty standard and can be found, along with axiomatic systems and proofs of soundness and completeness, in [14, 15], and [2], for instance.

We now move to accounting for the value part of the system. The philosophical underlying intuition is that, for each state $s$, if it exists, $\mu$ picks out the best (positive or negative) states agent $\alpha$ is prepared to bring about to preserve or preclude $s$.

The imprecise value assigned by an agent $\alpha$ to a proposition is now the collection of the states picked out by all the measures of the states exactly verifying that proposition.

We now define the imprecise value of a proposition $p$, for agent $k$, i.e. $V_{k}(p)$ in the following way:

Definition 13 (Imprecise value). Let $V_{k}(p)$, the imprecise value an agent $k$ attributes to a proposition $p$, be $V_{k}(p)=\bigcup_{s \in S}\left\{\bigcup_{i \in I} \mu_{i}^{k}(s): s \Vdash p\right\}$, where $\mu_{i}^{k} \in \mathcal{M}$ are the measures for an agent $k \in A$, with $i \in I$ an index.

Not only situations that occur can be evaluated: not all states are actual, yet, they can still verify or falsify propositions (cf. possible worlds). In fact, given the clauses for verification and falsification of a negated formula, it is possible to account for negative value, or the values of falsifiers.

Novel are the following clauses for a 'it is valued by agent $i$ at state $s$ ' operator, that intend to capture the intuition that a proposition is valued at a state $s$ in case there's a state $s^{\prime}$ that is valued from the original state which exactly makes true that proposition:

$s \Vdash-\nabla_{i} \phi$ iff there is an $s^{\prime}$, s.t. $s^{\prime} \Vdash \phi$ and $s^{\prime} \in V_{i}(\phi)$.

$s-\| \nabla_{i} \phi$ iff for all $s^{\prime} \Vdash \phi, s^{\prime} \notin V_{i}(\phi)$, or for some $s^{\prime}-\|, s^{\prime} \in V_{i}(\phi)$.

We call the present logic HVL (hyperintensional value logic). We now point out some results.

The resulting axiomatic system is one of those relatively well-known to track hyperintensionality, with the substitution of hyperintensional equivalents and with the addition of an axiom for the value operator that distributes over disjunction.

The axioms and rules are the following:

1. $A \approx_{H} A$

2. $A \approx_{H}(A \wedge A)$ 
3. $A \approx_{H}(A \vee A)$

4. $A \vee B \approx_{H}(B \vee A)$

5. $A \wedge B \approx_{H}(B \wedge A)$

6. $A \wedge(B \wedge C) \approx_{H}(A \wedge B) \wedge C$

7. $A \vee(B \vee C) \approx_{H}(A \vee B) \vee C$

8. $A \wedge(B \vee C) \approx_{H}(A \wedge B) \vee(A \wedge C)$

9. $A \approx_{H} \neg \neg A$

10. $(\neg A \wedge \neg B) \approx_{H} \neg(A \vee B)$

11. $(\neg A \vee \neg B) \approx_{H} \neg(A \wedge B)$

12. $(\neg A \vee \neg B) \approx_{H} \neg(A \wedge B)$

13. $\nabla_{i}(A) \vee \nabla_{i}(B) \approx_{H} \nabla_{i}(A \vee B)$

Rule:

1. $A \approx_{H} B, C(A) / C(B)$

THEOREM 4.2. HVL is sound and complete w.r.t. value space semantics.

Proof. The proof is similar to the soundness and completeness proof of $[13,2]$ with obvious variations.

We now highlight three useful facts.

FACT 4.3. It is not the case that if $\nabla_{i}(A) \wedge \nabla_{i}(B)$ then $\nabla_{i}(A \wedge B)$.

FACT 4.4. It is not the case that if $\nabla_{i}(A \wedge B)$ then $\nabla_{i}(A) \wedge \nabla_{i}(B)$.

Both results seem in line with a plausible notion of value: valuing $A$ and valuing $\mathrm{B}$ does not imply valuing $\mathrm{A}$ and $\mathrm{B}$, for $\mathrm{A}$ and $\mathrm{B}$, as we have discussed in the informal part, can interact in non-aggregative ways, perhaps decreasing the overall value. Conversely, valuing $\mathrm{A}$ and $\mathrm{B}$ does not imply valuing $A$ and valuing $B$ for an analogous reasoning.

Moreover, "conflicts of value" are not ruled out, consistently with the common occurrence of valuing opposite things:

FACT 4.5. $\forall \neg \neg\left(\nabla_{i}(A) \wedge \nabla_{i}(\neg A)\right)$. 
Logics of (dis)agreement We now introduce an operator that describe the notion of partial imprecise weak (dis)agreement among agents $i, \ldots, j \in$ $I$, namely an operator for weak agreement $\Delta_{i, \ldots, j}$, with $i, \ldots, j \in I$ and $|I|>1$ as follows:

$$
\begin{aligned}
& s \| \Delta_{i, \ldots, j} \phi \text { iff for all } s^{\prime} \text { s.t. } s^{\prime} \Vdash \phi, s^{\prime} \in V_{i, \ldots, j}(\phi), \text { where } \\
& V_{i, \ldots, j}(\phi)={ }_{d f} V_{i}(\phi) \cap \ldots \cap V_{j}(\phi) \\
& s-\Delta_{i, \ldots, j} \phi \text { iff for some } s^{\prime} \Vdash \phi, s^{\prime} \notin V_{i, \ldots, j}(\phi), \text { or for some } s^{\prime}-\| \\
& \phi, s^{\prime} \in V_{i, \ldots, j}(\phi) .
\end{aligned}
$$

We can now replace the axiom for value in HVL with the following axiom to obtain HLA, the hyperintensional logic of value agreement:

$$
\text { 14. } \Delta_{i, \ldots, j}(A \vee B) \approx_{H} \Delta_{i, \ldots, j} A \wedge \Delta_{i, \ldots, j} B
$$

THEOREM 4.6. HLA is sound and complete w.r.t. the above semantics.

Proof. The proof is similar to the soundness and completeness proof of $[13,2]$ with obvious variations.

We have an obvious bridge principle between the "it is valued" operator and the (dis)agreement operator:

$$
\mathrm{BP} \Delta_{i, \ldots, j}(\phi) \rightarrow_{H} \nabla_{i} \phi \wedge \ldots \wedge \nabla_{j} \phi
$$

where $\rightarrow_{H}$ is just one half of $\approx_{H}$. The converse of course does not hold, given the semantic clauses for conjunction and the (dis)agreement operator.

\section{Conclusion, limitations, and further work}

Building on the idea of understanding value and value disagreement using imprecise measures, a very essential sketch of a hyperintensional logic of value and disagreement, based on truthmaker semantics, has been given. Except for the conciseness of the sketch offered, there are some limitations and room for further research. First, more meta-theoretic results need to be proved. Second, more notions of disagreement needs to be defined precisely and formally modeled. Third, more bridge principles need to be studied and discussed with an eye to their philosophical significance. 
Acknowledgements. Many thanks to an anonymous reviewer and to many people who discussed some of the ideas behind this paper over the years in multiple places around the world, but first to Alberto Bardi, who patiently listened to very confused ramblings during a bike ride in the torrid summer of 2017. This work has been done while a postdoctoral research fellow of the Research Foundation, Flanders (FWO) and a Lise Meitner fellow (FWF), grant number M 2527 - G32.

\section{References}

[1] H. Andréka, M. Ryan, P.-Y. Schobbens, Operators and Laws for Combining Preference Relations, Journal of Logic and Computation, vol. 12(1) (2002), pp. 13-53, DOI: https://doi.org/10.1093/logcom/12.1.13.

[2] A. Anglberger, F. L. G. Faroldi, J. Korbmacher, An Exact Truthmaker Semantics for Obligation and Permission, [in:] O. Roy, A. Tamminga, M. Willer (eds.), Deontic Logic and Normative Systems, DEON16, College Publications, London (2016), pp. 16-31.

[3] R. J. Aumann, Utility Theory without the Completeness Axiom, Econometrica, vol. 30(3) (1962), pp. 445-462, DOI: https://doi.org/10.2307/1909888.

[4] F. Berto, D. Nolan, Hyperintensionality, [in:] E. N. Zalta (ed.), The Stanford Encyclopedia of Philosophy, spring 2021 ed., Metaphysics Research Lab, Stanford University (2021).

[5] R. Chang, Parity, Interval Value, and Choice, Ethics, vol. 115(2) (2005), pp. 331-350, DOI: https://doi.org/10.1086/426307.

[6] R. Chang, Value Incomparability and Incommensurability, [in:] I. Hirose, J. Olson (eds.), The Oxford Handbook of Value Theory, Oxford University Press (2015), DOI: https://doi.org/10.1093/oxfordhb/ 9780199959303.013.0012.

[7] R. Chang, Parity: An Intuitive Case, Ratio, vol. 29(4) (2016), pp. 395-411.

[8] C. Constantinescu, Value Incomparability and Indeterminacy, Ethical Theory and Moral Practice, vol. 15(1) (2012), pp. 57-70, DOI: https://doi.org/10.1007/s10677-011-9269-8.

[9] M. J. Cresswell, Hyperintensional Logic, Studia Logica, vol. 34(1) (1975), pp. 25-38, DOI: https://doi.org/10.1007/BF02314421. 
[10] F. Dietrich, C. List, What Matters and How It Matters: A Choice-Theoretic Representation of Moral Theories, The Philosophical Review, (2017), pp. 421-479, DOI: https://doi.org/10.1215/00318108-4173412.

[11] L. Elkin, G. Wheeler, Resolving Peer Disagreements Through Imprecise Probabilities, Nous, vol. LII(2) (2018), pp. 260-278, DOI: https: //doi.org/10.1111/nous.12143.

[12] F. L. G. Faroldi, Modeling Value Disagreement via Imprecise Measures, 2017.

[13] F. L. G. Faroldi, Hyperintensionality and Normativity, Springer, Dordrecht (2019), DOI: https://doi.org/10.1007\%2F978-3-030-03487-0.

[14] K. Fine, Angellic Content, Journal of Philosophical Logic, vol. 45(2) (2016), pp. 199-226, DOI: https://doi.org/10.1007/s10992-015-9371-9.

[15] K. Fine, Truthmaker Semantics, [in:] B. Hale, C. Wright, A. Miller (eds.), A Companion to the Philosophy of Language, 2nd ed., Blackwell, London (2017), pp. 556-577.

[16] J. Gert, Value and Parity, Ethics, vol. 114(3) (2004), pp. 492-510, DOI: https://doi.org/10.1086/381697.

[17] J. Gert, Parity, Preference and Puzzlement, Theoria, vol. 81(3) (2015), pp. 249-271, DOI: https://doi.org/10.1111/theo.12069.

[18] C. Hare, Take the Sugar, Analysis, vol. 70(2) (2010), pp. 237-247, DOI: https://doi.org/10.1093/analys/anp174.

[19] W. Krysztofiak, Algebraic Models of Mental Number Axes: Part II, Axiomathes, vol. 26(2) (2016), pp. 123-155, DOI: https://doi.org/10.1007/ s10516-015-9270-2.

[20] W. Krysztofiak, Representational Structures of Arithmetical Thinking: Part I, Axiomathes, vol. 26(1) (2016), pp. 1-40, DOI: https://doi.org/ 10.1007/s10516-015-9271-1.

[21] H. Leitgeb, HYPE: A System of Hyperintensional Logic (with an Application to Semantic Paradoxes), Journal of Philosophical Logic, vol. 48(2) (2019), pp. 305-405, DOI: https://doi.org/10.1007/s10992-018-9467-0.

[22] D. McCarthy, K. Mikkola, T. Thomas, Aggregation for General Populations Without Continuity or Completeness, [in:] MPRA Paper No. 80820, University Library of Munich, Germany (2017).

[23] D. McCarthy, K. Mikkola, T. Thomas, Representation of Strongly Independent Preorders by Sets of Scalar-Valued Functions, [in:] MPRA Paper No. 79284, University Library of Munich, Germany (2017). 
[24] D. McCarthy, K. Mikkola, T. Thomas, Representation of Strongly Independent Preorders by Vector-Valued Functions, [in:] MPRA Paper No. 80806, University Library of Munich, Germany (2017).

[25] W. Rabinowicz, Value Relations, Theoria, vol. 74(1) (2008), pp. 18-49, DOI: https://doi.org/10.1111/j.1755-2567.2008.00008.x.

[26] W. Rabinowicz, I-Wlodek Rabinowicz: Incommensurability and Vagueness, Aristotelian Society Supplementary Volume, vol. 83(1) (2009), pp. 71-94, DOI: https://doi.org/10.1111/j.1467-8349.2009.00173.x.

[27] R. Suszko, An essay in the formal theory of extension and of intension, Studia Logica, vol. 20(1) (1967), pp. 7-34, DOI: https://doi.org/10.1007/ BF02340023.

[28] L. S. Temkin, Rethinking the Good: Moral Ideals and the Nature of Practical Reasoning, Oxford Ethics, Oxford University Press (2012), DOI: https://doi.org/10.1093/acprof:oso/9780199759446.001.0001.

[29] V. Torra, Y. Narukawa, M. Sugeno (eds.), Non-Additive Measures. Theory and Applications, Springer (2014), DOI: https://doi.org/10.1007/ 978-3-319-03155-2.

[30] B. C. van Fraassen, Facts and Tautological Entailment, Journal of Philosophy, vol. 66(15) (1969), pp. 477-487, DOI: https://doi.org/10.2307 /2024563.

\section{Federico L. G. Faroldi}

Research Foundation, Flanders (FWO)/Ghent University

Department of Philosophy

Blandijnberg 2 \#9000

Ghent, Belgium

e-mail: federico.faroldi@ugent.be 